\title{
MANGANESE(III) ACETATE AND CERIUM(IV) AMMONIUM NITRATE MEDIATED OXIDATIVE RADICAL ADDITIONS OF $\beta$-DIKETONES AND $\beta$-KETOESTERS TO LIMONENE: CHEMO AND REGIOSPECIFIC PREPARATION OF 2,3-DIHYDROFURANS
}

\author{
Marcio C.S. de Mattos*a, Soraia P.L. de Souza ${ }^{a}$ and Simone M. Elias ${ }^{\mathrm{b}}$ \\ ${ }^{a}$ Instituto de Quimica, Departamento de Quimica Orgânica, \\ Universidade Federal do Rio de Janeiro, Caixa Postal 68545, \\ 21945-970, Rio de Janeiro, Brazil \\ ${ }^{b}$ Instituto de Quimica, Universidade do Eistado do Rio de Janeiro, Rio de Janeiro, Brazil
}

\begin{abstract}
Mn}(\mathrm{OAc})_{3} \cdot 2 \mathrm{H}_{2} \mathrm{O}$ mediated oxidative radical addition of $\beta$-diketones (acetylacetone, benzoylacetone, 1,3cyclohexanodione) and $\beta$-ketoesters (ethyl acetoacetate, ethyl benzoylacetate) to limonene produced chemo- and regiospecificaly 2,3-dihydrofurans as a ca. 1:1 mixture of diastereoisomers in $72-86 \%$ isolated yields. On the other hand, the reactions mediated by CAN produced the same products in lower yields (29-35\%).
\end{abstract}

\section{Introduction}

The addition of organic free radicals to alkenes is a powerful methodology for construction of C-C bonds (1) and among them the so-called "oxidative free radical addition" mediated by transition metal salts has enormously increased in the last 30 years (2). Examples of the synthetic utility of this methodology are the preparation of $\delta$ bulyrolactones and 2,3-dihydrofunas by the reactions of alkenes with carboxylic acids and $\beta$-dicarbonyl compounds, respectively (3).

We reported (4) the chemo- and regiospecific formation of the natural product norbisabolide $\left(2, R_{1}=R_{2}=H\right)$ and related $\delta$-butyrolactones in good yields by the oxidative radical addition of carboxylic acids to limonene 1 mediated by CAN [cerium(IV) ammonium nitrate]. On the other hand, a group from India (5) published a similar reaction using $\beta$-diketones (dimedone or acetylacetone), producing the 2,3-dihydrofurans $\underline{3}$ in moderate yield (Scheme 1). Recently (6) we investigated the addition of ethyl aceloacetate to limonene and the results were disappointing with $\mathrm{Ce}(\mathrm{IV}), \mathrm{Fe}$ (III), $\mathrm{Co}(\mathrm{II})$, and $\mathrm{Cu}(\mathrm{II})$, but good when $\mathrm{Mn}(\mathrm{OAc})_{3} \cdot 2 \mathrm{H}_{2} \mathrm{O}$ was employed.

\footnotetext{
* to whom correspondence should be addressed. E-mail: mmallos@iq.ufrj.br
} 
<smiles>C=C(C)C1CC=C(C)CC1</smiles>

Scheme 1

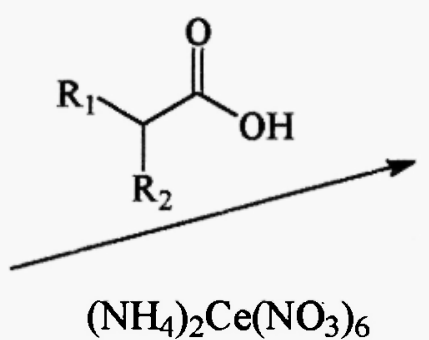<smiles>[R]C(=O)CC(C)=O</smiles>

Continuing our interest in chemo- and regiospecific reactions of limonene $(4,6,7)$, we describe here our results on the preparation of 2,3-dihydrofurans by reaction of limonene with diverse $\beta$-diketones and $\beta$-ketoesters mediated by $\mathrm{CAN}$ and by $\mathrm{Mn}(\mathrm{OAc})_{3} \bullet 2 \mathrm{H}_{2} \mathrm{O}$.

\section{Results}

The reactions were performed stirring limonene $(10 \mathrm{mmol})$ with the $\beta$-dicarbonyl compounds $(10 \mathrm{mmol})$ in the presence of CAN (20 mmol) to produce the corresponding 2,3-dihydrofurans in $29-35 \%$ isolated yield as a $c a$. $1: 1$ mixture of diastereoisomers (8) - detern ned by high resolution gas chromatography. As the yields were low, we revisited the reaction using $\left.\mathrm{Mn}(\mathrm{CAc})_{3} \cdot 2 \mathrm{H}_{2}\right)$ in the place of $\mathrm{CAN}$ and obtained the same products but in higher isolated yields (72-86\%) - Scheme 2 and Table 1 summarize the results.

In summary, in contrast with the literature information (3a, 5, 9), in our hands, $\mathrm{Mn}(\mathrm{OAc})_{3} \cdot 2 \mathrm{H}_{2} \mathrm{O}$ was better than CAN to mediate the oxidative radical addition of $\beta$-dicarbonyl compounds to limonene. Furthermore the utilization of the $\mathrm{Mn}$ (III) salt is also very convenient because it is a superior reagent in terms of atom economy (10) (its molecular weigh is $c a$. half of the CAN to produce the same effect, $i e$, one electron transferring) 


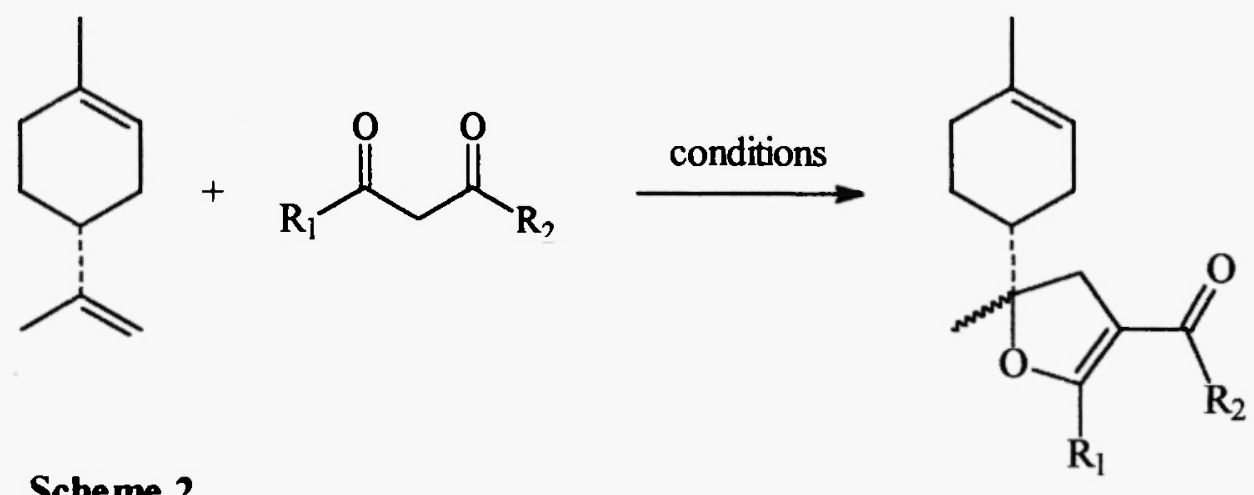

\section{Scheme 2}

\begin{tabular}{|c|c|c|c|}
\hline $\mathbf{R}_{1}$ & $\mathbf{R}_{2}$ & Conditions & Yield (\%) $^{\mathbf{A}}$ \\
\hline $\mathrm{Me}$ & $\mathrm{Me}$ & $\mathrm{CAN} / \mathrm{MeOH} / 5^{\circ} \mathrm{C} / 90 \mathrm{~min}$ & 29 \\
\hline $\mathrm{Me}$ & $\mathrm{Me}$ & $\mathrm{Mn}(\mathrm{OAC})_{3} \bullet 2 \mathrm{H}_{2} \mathrm{O} / \mathrm{HOAc} / 70^{\circ} \mathrm{C} / 90 \mathrm{~min}$ & 86 \\
\hline $\mathrm{Me}$ & $\mathrm{Ph}$ & $\mathrm{CAN} / \mathrm{MeOH} / 5^{\circ} \mathrm{C} / 90 \mathrm{~min}$ & 35 \\
\hline $\mathrm{Me}$ & $\mathrm{Ph}$ & $\mathrm{Mn}(\mathrm{OAc})_{3} \bullet 2 \mathrm{H}_{2} \mathrm{O} / \mathrm{HOAc} / 70^{\circ} \mathrm{C} / 90 \mathrm{~min}$ & 80 \\
\hline \multicolumn{2}{|c|}{$-\left(\mathrm{CH}_{2}\right)_{3^{-}}$} & $\mathrm{Mn}(\mathrm{OAc})_{3} \bullet 2 \mathrm{H}_{2} \mathrm{O} / \mathrm{HOAc} / 70^{\circ} \mathrm{C} / 120 \mathrm{~min}$ & 86 \\
\hline $\mathrm{Mc}$ & $\mathrm{OEt}$ & $\mathrm{CAN} / \mathrm{McOH} / 5^{\circ} \mathrm{C} / 20 \mathrm{~min}$ & 35 \\
\hline $\mathrm{Me}$ & $\mathrm{OEt}$ & $\mathrm{Mn}(\mathrm{OAc})_{3} \bullet 2 \mathrm{H}_{2} \mathrm{O} / \mathrm{HOAc} / 70^{\circ} \mathrm{C} / 90 \mathrm{nun}$ & 72 \\
\hline $\mathrm{Ph}$ & $\mathrm{OEt}$ & $\mathrm{Mn}(\mathrm{OAc})_{3} \bullet 2 \mathrm{H}_{2} \mathrm{O} / \mathrm{HOAc} / 70^{\circ} \mathrm{C} / 90 \mathrm{~min}$ & 76 \\
\hline
\end{tabular}

aisolated yield.

Table 1. Yields of 2,3-dihydrofurans from the reaction of limonene and diverse $\beta$-dicarbonyl compounds mediated by $\mathrm{CAN}$ and by $\mathrm{Mn}(\mathrm{OAc})_{3} \cdot 2 \mathrm{H}_{2} \mathrm{O}$.

\section{Acknowledgements}

SPLS and SME thank PIBIC/UFRJ and CETREINA/UERJ, respectively, for fellowships. We thank Prof. W.Bruce Kover and Joel Jones Jr (IQ/UFRJ) for helpful discussions.

\section{References and notes}

(1) (a) B. Giese. Angew. Chem. Int. Ed Engl. 24, 553 (1985). (b) D.P. Curran, Synthesis 417, 489 (1988). (c) C.P. Jasperse, D.P. Curran and T.L. Fevig, Chem. Rev. 91, 1237 (1991). (d) F. Aldabbagh and W.R. Bowman, Contemp. Org. Synth. 4, 261 (1997).

(2) (a) G.G. Melikyan, Synthesis 833 (1993). (b) V. Nair, J. Mathew and J. Prabhakaran, Chem. Soc. Rev. 26, 127 (1997). (c) J. Iqbal, B. Bhatia and N.K. Nayyar, Chem. Rev. 94, 519 (1994). 
(3) Recent examples: (a) S. Muthusamy, C. Gunanathan and S.A. Babu, Synlett 787 (2002). (b) S.B. Solabannavar, V.B. Helavi, U.V. Desai and R.B. Mane, Tetrahedron Lett. 43, 4535 (2002). (c) M. Yilmaz and A.T. Pekel, Synth. Commun. 31, 3871 (2001).

(4) M.C.S. de Mattos and S.M. Elias, J. Braz. Chem. Soc. 6, 377 (1995).

(5) V. Nair, J. Mathew and L.G. Nair, Synth. Commun. 26, 4531 (1996).

(6) M.C.S. de Mattos, S.P.L. de Souza and S.M. Elias, Heterocycl. Commun. 7, 249 (2001).

(7) A.M. Sanseverino, M.C.S. de Mattos and W.B. Kover, J. Chem. Res. 346 (2000) and references cited therein.

(8) Selected analytical data. (I'R,5RS)-3-ucetyl-2,5-dimethyl-5-(4'-methylcyclohex-3'-enyl)-4,5-dihydrofuran (mixture of diastereoisomers): ${ }^{2} \mathrm{H}$ NMR (200 MHz, $\mathrm{CDCl}_{3}$ ): $\delta 5.20$ (brs, $1 \mathrm{H}$, olefinic), 2.90-2.32 (m, 3H), 2.15 (s, 6H), 1.90$1.70(\mathrm{~m}, 6 \mathrm{H}), 1.60(\mathrm{~s}, 3 \mathrm{H}), 1.22(\mathrm{~s}, 3 \mathrm{H}) \mathrm{ppm} .{ }^{13} \mathrm{C}$ NMR (75 MHz, CDCl 3$): \delta 193.8(\mathrm{C}=\mathrm{O}), 166.0(\mathrm{q}), 132.5(\mathrm{q})$, $120.2(\mathrm{CH}), 112.4$ (q), 91.0, 90.7 (q), 42.5, $42.3(\mathrm{CH}), 39.2,39.0\left(\mathrm{CH}_{2}\right), 30.3,30.1\left(\mathrm{CH}_{2}\right), 29.1\left(\mathrm{CH}_{2}\right), 26.0,25.4$ $\left(\mathrm{CH}_{3}\right), 24.0,23.8\left(\mathrm{CH}_{3}\right), 23.0\left(\mathrm{CH}_{3}\right), 15.0\left(\mathrm{CH}_{3}\right) \mathrm{ppm}$. MS (70 eV, electron impact): $\mathrm{m} / \mathrm{z} 234,216,191,173,151$, 134 (100\%), 119, 93, 79. (1'R,2RS)-2-methyl-2-(4'-methylcyclohex-3'-enyl)-2,3,5,6,7-pentahydrobenzo[1,2b/furan-4-one (mixture of diastereoisomers): ${ }^{1} \mathbf{H}$ NMR (200 MHz, $\mathrm{CDCl}_{3}$ ): $\delta 5.30$ (brs, 1H, olefinic), 2.60-2.30 (m, $4 \mathrm{H}), 2.20(\mathrm{~s}, 3 \mathrm{H}), 2.10(\mathrm{~s}, 2 \mathrm{H}), 1.95-1.64(\mathrm{~m}, 6 \mathrm{H}), 1.50(\mathrm{~s}, 3 \mathrm{H}), 1.30(\mathrm{~s}, 3 \mathrm{H}) \mathrm{ppm} .{ }^{13} \mathrm{C}$ NMR (75 MHz, $\left.\mathrm{CDCl}_{3}\right)$ : $195.1(\mathrm{C}=\mathrm{O}), 175.2$ (q), 133.9 (q), $120.0(\mathrm{CH}), 111.2$ (q), 95.1, 94.9 (q), 42.7, $42.5(\mathrm{CH}), 38.1\left(\mathrm{CH}_{2}\right), 34.6\left(\mathrm{CH}_{2}\right)$, $34.0\left(\mathrm{CH}_{2}\right), 33.5\left(\mathrm{CH}_{2}\right), 29.9\left(\mathrm{CH}_{2}\right), 28.3,28.1\left(\mathrm{CH}_{2}\right), 25.9,25.6\left(\mathrm{CH}_{2}\right), 24.0,23.7\left(\mathrm{CH}_{3}\right), 22.2\left(\mathrm{CH}_{3}\right)$ ppm $\mathrm{MS}(70$ $\mathrm{eV}$, electron impact): $\mathrm{m} / \mathrm{z} 246,228,217,163,151,134,121$ (100\%), 119, 93, 79. (1'R,5RS)-3-carboethoxy-2,5dimethyl-5-(4'-methylcyclohex-3'-enyl)-4,5-dihydrofuran (mixture of diastereoisomers): ' $\mathbf{H}$ NMR (200 MHz, $\mathrm{CDCl}_{3}$ ): $\delta 5.38$ (brs, $1 \mathrm{H}$, olefinic), 4.16 (q, $2 \mathrm{H}$ ), 2.82 (d, $\left.1 \mathrm{H}\right), 2.46$ (dd, $\left.1 \mathrm{H}\right), 2.17$ (s, 3H), 1.98-1,73 (m, 7H), 1.65 (s, 3H), 1.30 (s, 3H), 1.29 (s, 3H) ppm. ${ }^{13} \mathrm{C}$ NMR (75 MHz, $\left.\mathrm{CDCl}_{3}\right): \delta 167.2,167.0(\mathrm{C}=\mathrm{O}), 166.8$ (q), 134.3, 134.1 (q), 120.3, $120.2(\mathrm{CH}), 101.2(\mathrm{q}), 90.8,90.6(\mathrm{q}), 59.5\left(\mathrm{CH}_{2}\right), 43.3,43.2(\mathrm{CH}), 39.2,38.5\left(\mathrm{CH}_{2}\right), 30.7,30.6\left(\mathrm{CH}_{2}\right), 29.9$ $\left(\mathrm{CH}_{2}\right), 24.9,24.5\left(\mathrm{CH}_{3}\right), 23.7\left(\mathrm{CH}_{3}\right), 14.7,14.5\left(\mathrm{CH}_{3}\right)$ ppm. MS (70 eV, electron impact): $\mathrm{m} / \mathrm{z} 264,246,219,191$, $172,157,134(100 \%), 119,97,79$.

(9) (a) Y.R. Lee, B.S. Kim and D.H. Kim, Tetrahedron 56, 8845 (2000). (b) V. Nair, J. Mathew and K.V. Radhakrishnan. J. Chem. Soc. Perkin Trans. 11487 (1996).

(10) (a) B.M. Trost, Science 254, 1471 (1991). (b) A.M. Sanseverino, Quim. Nova 23, 102 (2000).

\section{Received on January 22, 2003}

